\title{
Request for Public Comment on High Performance Fortran
}

\author{
CHARLES KOELBEL
}

CITC-CRPC, Rice University, Houston, TX 772.51

\section{TO: THE HIGH PERFORMANCE COMPUTING COMMUNITY}

\section{Invitation}

The High Performance Fortran Forum (HPFF). with participation from over 40 organizations. has been meeting for the last 10 months to discuss and define a set of extensions to Fortran called High Performance Fortran (HPF). Our goal is to address the problems of writing data parallel programs for architectures where the distribution of data imparts performance. Although we hope that the HPF extensions become widely available. HPFF is not sanctioned or supported by any official standards organization. At this time. HPFF invites general public review comments on the initial version of the language draft.

The HPF language specification. version 1.0. will be available after mid December. This version is primarily an editorial improvement of previous drafts, based on suggestions from public comment.

HPFF invites comments on the technical content of $\mathrm{HPF}$, as all as on the editorial presentation in the document.

\section{How to Get the Documents}

Electronic copies of the HPF language specification are available from numerous sources.

\author{
Anonymous FTP sources: \\ titan.cs.rice.edu \\ think.com \\ lip.gmd.de \\ theory.tc.cornell.edu \\ minerva.npacesyredu \\ Email sourres: \\ netlib@ research.ait.com \\ netib@oml.gov \\ softlib@es.rice.edu
}

\author{
Directory: \\ public/HPFF/draft \\ pullic/HPFF \\ hpt-europe \\ put \\ public \\ First line of message: \\ send hpf-v10.ps from hplf \\ send hpl-r10.ps from hpl \\ send hpl-r10.ps
}

Rereived Renember 1902

(C) 1093 by John Wiley s Sons. Ine.

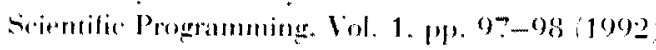

(c: (: $10.58-92++/ 93 /(2) 20095-6) 2$

The following formats are available. Note that not all formats are available from all sources. 


$$
\begin{aligned}
& \text { hpf-v10.dvi } \\
& \text { hpf-v10.ps } \\
& \text { hpf-v10.ps.Z } \\
& \text { hpf-v10.tar } \\
& \text { hpf-v10.tar.Z }
\end{aligned}
$$

Dil file

Postscript

Compressed Postscript

Tar file of LaTeX version

Compressed tar file
For more detailed instructions, send email to hpff-info@cs.rice.edu. This will return a message with expanded detail about accessing the above. document sources, as well as other information about HPFF.

We strongly encourage reviewers to obtain an electronic copy of the document. However. if electronic access is impossible the draft is also available in hard copy form as CRPC Technical Report \#92225. This report is available for $\mathrm{S} 50$ (copving/handling fee) from: Theresa Chatman. CITI/ CRPC. Box 1892. Rice Lniversity, Houston, TX 77251. Make checks pavable to Rice Lniversity. This document will be sent surface mail unless additional airmail postage is included in the payment.

\section{How to Submit Comments}

HPFF encourages reviewers to submit comments as soon as possible. Please do not submit com- ments for any version of the draft earlier than the 0.4 version.

Please send comments by email to hplf-comments@cs.rice.edu. To facilitate the processing of comments we request that separate comment messages be submitted for each chapter of the document and that the chapter be clearly identified in the "Subject:" line of the message. Comments about general overall impressions of the HPF document should be labeled as Chapter 1 . All comments on the language specification become the property of Rice Lniversity.

If email access is impossible for comment re-sponses, hard copy may be sent to: HPF Comments. c/o Theresa Chatman. CITI/CRPC. Box 1892. Rice Lniversity. Houston. TX 7:251.

HPFF plans to process the feedback received at a meeting in March. Best efforts will be made to reply to comments submitted. 

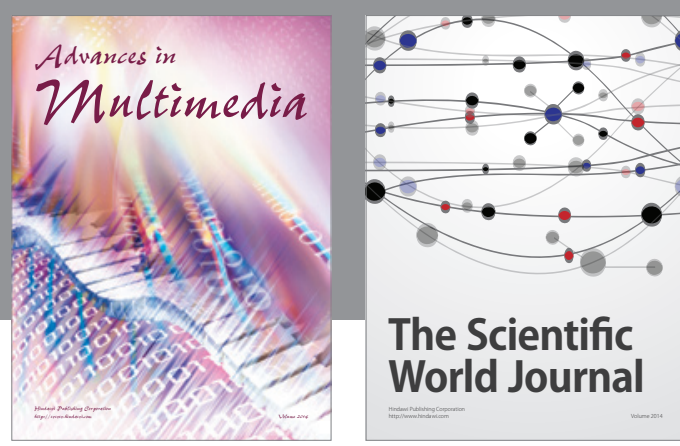

The Scientific World Journal
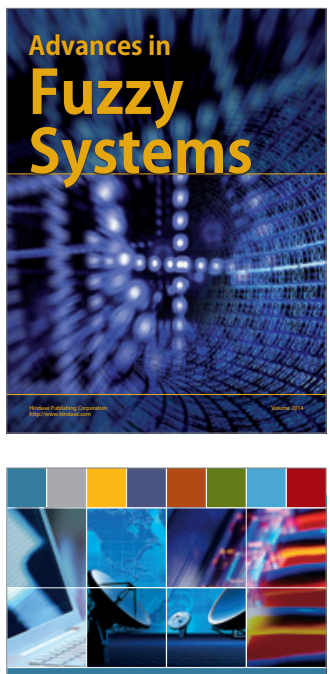

Computer Networks and Communications
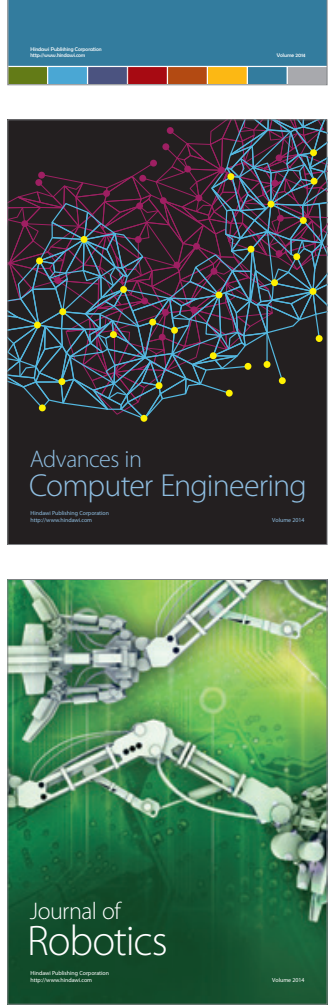
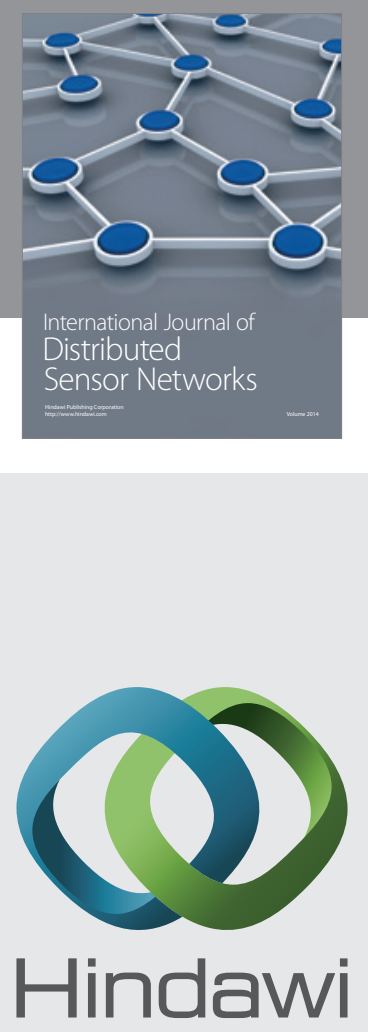

Submit your manuscripts at

http://www.hindawi.com
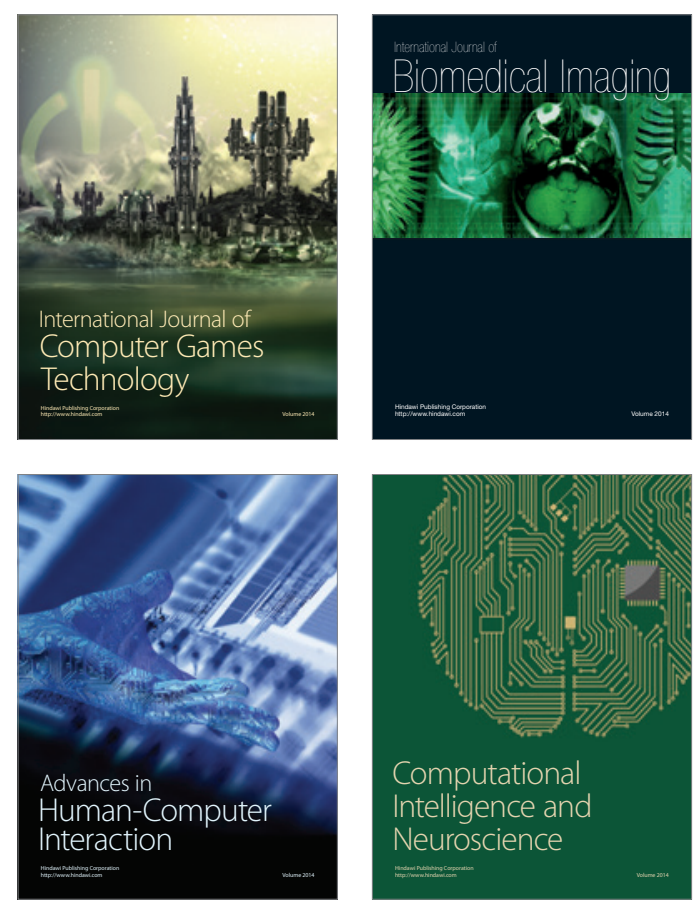
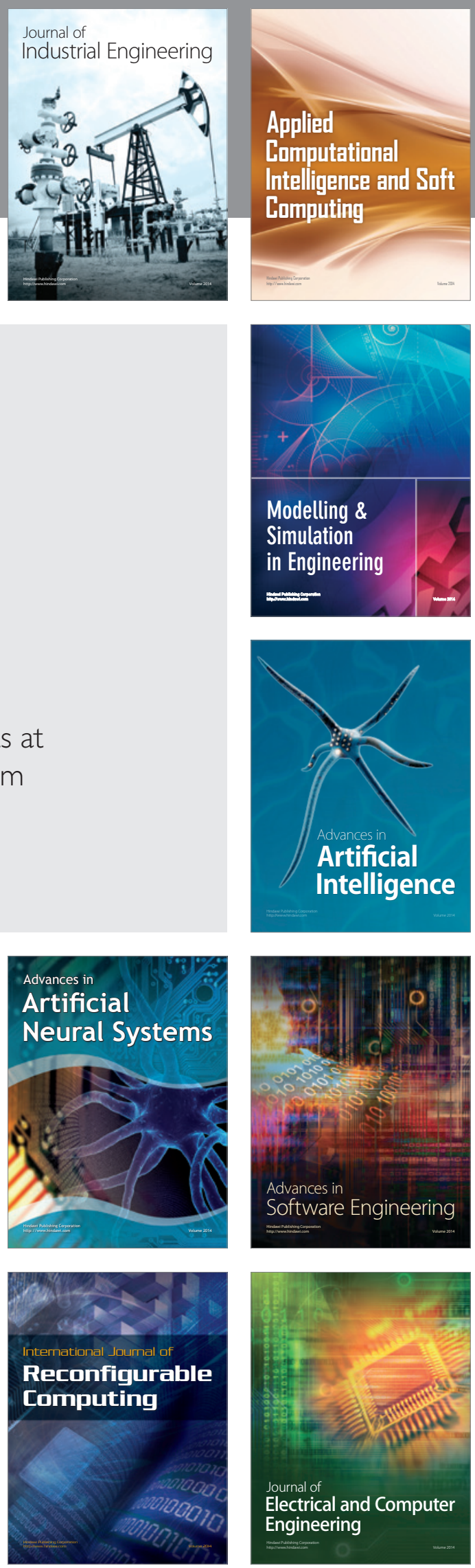